\title{
The drug tolerant persisters of Riemerella anatipestifer can be eradicated by a combination of two or three antibiotics
}

\author{
Tian Tang ${ }^{1} \mathbb{D}$, Yanxia Wu' ${ }^{1}$, Hua Lin², Yongyu Li ${ }^{1}$, Haojiang Zuo ${ }^{1}$, Qun Gao ${ }^{3}$, Chuan Wang ${ }^{1 *}$ and Xiaofang Pei ${ }^{1^{*}}$
}

\begin{abstract}
Background: Riemerella anatipestifer (RA), the causative agent of duck infectious serositis, leads to high mortality in duck flocks and great economic losses in duck industry. Previous studies on RA are largely focused on its detection, virulence factors, serology, epidemiology as well as antibiotic resistance. Neither drug tolerant persisters nor the persister level under the treatment of antibiotics has been revealed. The persisters are non-growing or dormant cells within an isogenic bacterial population; they play important roles in recurrent infection and formation of drug resistant mutants. The aim of this study is to detect the drug tolerant persisters from the exponentially grown population of RA reference strain (RA 11845) or RA clinical isolate (RA TQ3), and address whether a single antibiotic or a combination of two or three antimicrobials can eradicate the persisters at respective maximum serum/plasma concentration $\left(C_{\max }\right)$.

Result: With the concentration of a test antibiotic increased, a small fraction of cells in the exponentially grown culture of RA reference strain (RA 11845) or RA clinical isolate (RA TQ3) always survived, irrespective of treatment time, indicating the presence of drug tolerant presisters. A single antibiotic cannot eradicate the persisters of both RA strains at respective $C_{\max }$ except that the $C_{\max }$ of ceftiofur wiped out the population of the reference strain (RA 11845). Besides, the clinical isolate RA TQ3 presented a higher tolerance to ceftiofur in comparison to that of the reference strain (RA 11845). Combination of any two or three antimicrobials eliminated the drug tolerant persisters of RA TQ3 completely at respective $C_{\max }$.
\end{abstract}

Conclusion: A sub-community of drug tolerant persisters was present in RA population. Persisters of RA TQ3 are single drug tolerant and not multidrug tolerant persisters.

Keywords: Riemerella anatipestifer, Persisters, Antibiotics, Drug tolerance

\section{Background}

Duck meat has traditionally been an important source of animal protein in many Asian countries; world production was approximately 4.4 million tons in 2013 [1]. Infectious diseases are therefore of increasing interest to the duck industry. Riemerella anatipestifer (RA), a Gram-negative, nonmotile, spore-forming, and rod-shaped bacterium, is the etiological agent of duck exudative septicemia or infectious serositis, causing great economic losses in duck industry worldwide due to high mortality (up to $75 \%$ ),

\footnotetext{
* Correspondence: wangchuan@scu.edu.cn; xxpei@scu.edu.cn

${ }^{1}$ Department of Public Health Laboratory Sciences, West China School of Public Health, Sichuan University, 16\#, Section 3, South Renmin Road, Chengdu, Sichuan 610031, People's Republic of China

Full list of author information is available at the end of the article
}

weight loss, and treatment cost [2-4]. Besides ducks, other poultry species such as chicken, geese, and turkeys are also susceptible to RA infection [5-8].

Previous work on RA mostly focused on its detection [9-11], virulence factors [12, 13], serology [14-16], epidemiology [17-19], and antibiotic resistance [20, 21]. Neither drug tolerant persisters nor the persister level of RA under the treatment of clinical antibiotics has been revealed. The persisters are non-growing or dormant cells within an isogenic bacterial population, capable of enduring lethal doses of antibiotics due to their inactive physiological state [22-24]. Unlike drug-resistant mutants with genetic change which can proliferate in the presence of antibiotics, persisters do not replicate in the same condition. The antibiotic tolerance of persisters is

(c) The Author(s). 2018 Open Access This article is distributed under the terms of the Creative Commons Attribution 4.0 International License (http://creativecommons.org/licenses/by/4.0/), which permits unrestricted use, distribution, and 
transient, non-genetic, and not inheritable [25]. Once drugs are removed, persisters restart growth and result in a population that is again antibiotic sensitive [26]. Although persister cells account for merely 0.001 to $1 \%$ of the entire bacterial population, they play important roles in recurrent infection and formation of drug resistant mutants [26]. In the present study, we sought to determine if persisters are present in RA population and address whether the maximum serum/plasma concentration $\left(C_{\max }\right)$ of a single antibiotic or a combination of two or three antimicrobials, each at $\mathrm{C}_{\max }$, can eradicate the persisters.

\section{Results}

Identification of persisters from exponential culture of RA Prior to investigating the drug tolerant persisters, we determined the MIC of each antibiotic for each RA strain. The results are summarized in Table 1 . To detect the persisters from exponential growing culture of RA reference strain (RA 11845) or RA clinical isolate (RA TQ3), we performed a time-dependent killing experiment, using three clinical antibiotics (ceftiofur, ciprofloxacin and spectinomycin). As shown in Fig. 1a, exposure to increasing concentrations of ceftiofur resulted in a rapid decrease in bacterial population of RA 11845, with a sharp reduction $>99.9 \%$ (the number of killed cells divided by the number of cells before addition of drug) after $24 \mathrm{~h}$ treatment at 10- and 80-fold MIC, followed by a steady value up to $48 \mathrm{~h}$, indicating the presence of ceftiofur-tolerant persisters. When ciprofloxacin was added to the culture in a final concentration of 80 -fold MIC, the bacterial number barely changed within the first $6 \mathrm{~h}$, then decreased drastically before reaching the plateau at $24 \mathrm{~h}$ (Fig. 1b). Similar biphasic killing curve was observed in the presence of 10-fold MIC of ciprofloxacin, except the time that reached the plateau was delayed to $27 \mathrm{~h}$ (Fig. 1b). These data suggest that ciprofloxacin-tolerant persisters existed in bacterial population of RA 11845. Spectinomycin was more efficient in killing the exponential growing cells of RA 11845 in comparison to ceftiofur and ciprofloxacin, since more than $99.9 \%$ of cells were sterilized by spectinomycin in the initial $12 \mathrm{~h}$, irrespective of drug dosage (Fig. 1c). The killing curves of spectinomycin also exhibited a biphasic pattern, indicating the presence of spectinomycin-tolerant persisters.
When exponential population of RA TQ3 was challenged with ceftiofur at 80 -fold MIC, the number of survivors decreased over time without showing a plateau on the killing curve (Fig. 1a). Besides, $73.2 \%$ of RA TQ3 cells were destroyed after $12 \mathrm{~h}$ of treatment in comparison to that of $99.8 \%$ of RA 11845 cells were abolished under a similar dosage (80-fold MIC). Collectively, this indicate that RA TQ3 are more tolerant to ceftiofur than the RA reference strain (RA 11845). Intriguingly, 10-fold MIC of ceftiofur diminished the population of RA TQ3 in the initial $6 \mathrm{~h}$, but the cell number increased thereafter, indicating the generation of ceftiofur-resistant mutants which are capable of proliferating in the presence of ceftiofur (Fig. 1d). The increasing concentrations of ciprofloxacin and spectinomycin alone killed the non-tolerant cells of RA TQ3 rapidly in the initial 18 and $12 \mathrm{~h}$, respectively, leaving a substantial number of survivors. The number of survivors barely changed over time, and showed a plateau on the killing curves of each drug (Fig. 1b \& c), indicating that these survivors were persisters. The killing curves of each drug for each RA strains at 20- and 40-fold MIC are presented in Additional file 1: Table S1.

To further validate that the surviving bacteria were in fact persisters and not resistant bacteria, we isolated the survivors of RA 11845 from $48 \mathrm{~h}$ of treatment with 80 -fold MIC of ceftiofur, ciprofloxacin, or spectinomycin. These survivors were regrown in fresh media to form a population in exponential phase, followed by the challenge with same antibiotic and dosage again to finish the first cycle. After three consecutive cycles, we did not observe an elevated level of survivors (Fig. 2a-c); the bacterial population derived from the survivors of each cycle was as sensitive to each antibiotic as the parental strain RA 11845. All of these evidences suggested that these survivors are persisters, because, their drug tolerance is transient and non-heritable.

\section{The persister level of RA under $C_{\max }$ of a single antibiotic} Given the potential of persisters to cause a relapse, it is important to address whether the $\mathrm{C}_{\max }$ of an antibiotic can eradicate the drug tolerant persisters. As shown in Fig. 2a-c, none of the tested antibiotic was able to eliminate persister cells of RA TQ3 alone at

Table 1 MIC of each antibiotic, $C_{\max }$ relative to MIC, and the $\mathrm{FICl}$ values of combining two drugs against RA 11845 or RA TQ3

\begin{tabular}{|c|c|c|c|c|c|c|c|c|c|c|c|c|}
\hline \multirow[t]{2}{*}{ Strains } & \multicolumn{3}{|c|}{ MIC $(\mu \mathrm{g} / \mathrm{ml})$} & \multicolumn{3}{|c|}{$\mathrm{C}_{\max } / \mathrm{MIC}$} & \multicolumn{6}{|l|}{$\mathrm{FICls}$} \\
\hline & CEF & CIP & SPE & CEF & CIP & SPE & $\mathrm{CEF}+$ & & CEF & & $\mathrm{CIP}+$ & \\
\hline RA 11845 & 0.03125 & 0.025 & 20.0 & 420 & 189 & 8 & 0.75 & (AD) & 1.0 & (AD) & 0.56 & (AD) \\
\hline RA TQ3 & 0.0625 & 1.0 & 40.0 & 210 & 5 & 4 & 0.75 & (AD) & 1.0 & (AD) & 1.0 & (AD) \\
\hline
\end{tabular}

$\mathrm{CEF}=$ Ceftiofur; $\mathrm{CIP}=$ Ciprofloxacin; SPE = Spectinomycin 


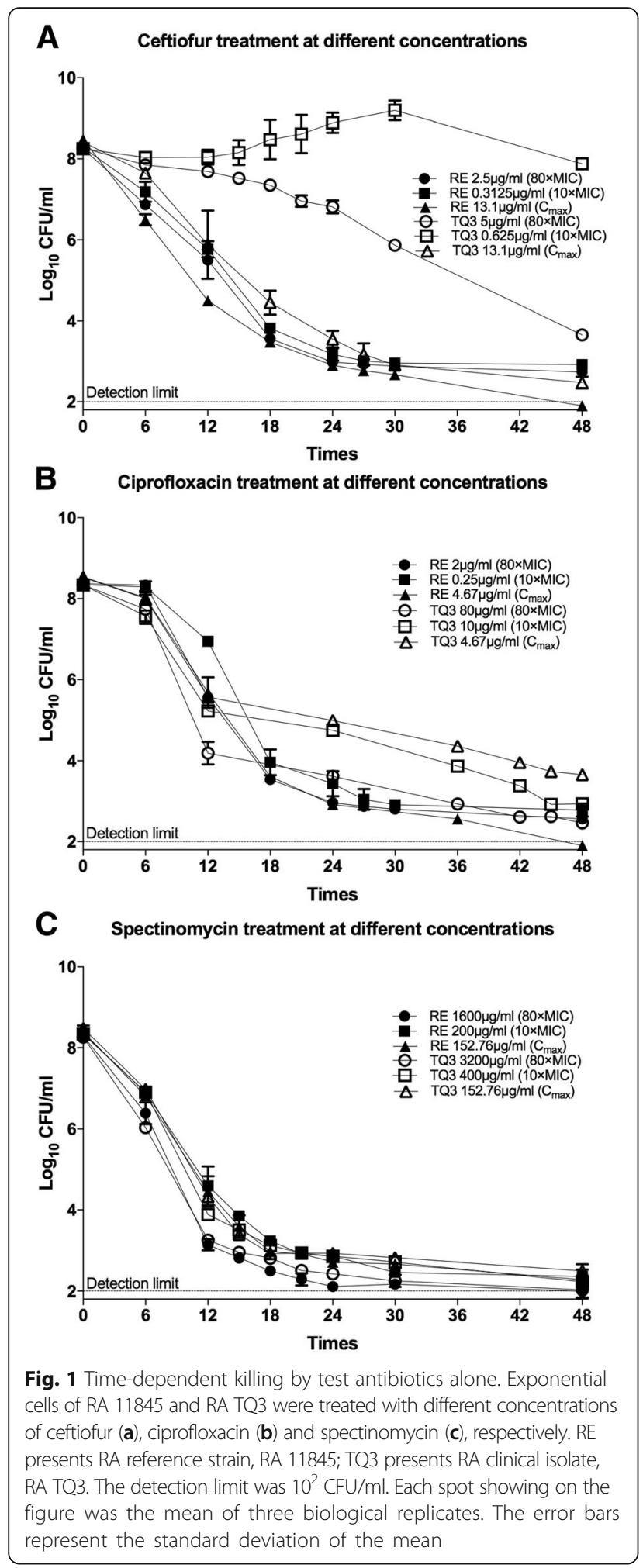

$\mathrm{C}_{\max }$. Similar results were observed in exponentially grown population of RA 11845 , except that the $C_{\max }$ of ceftiofur wiped out all bacterial cells within a period of $48 \mathrm{~h}$ (Fig. 2a).

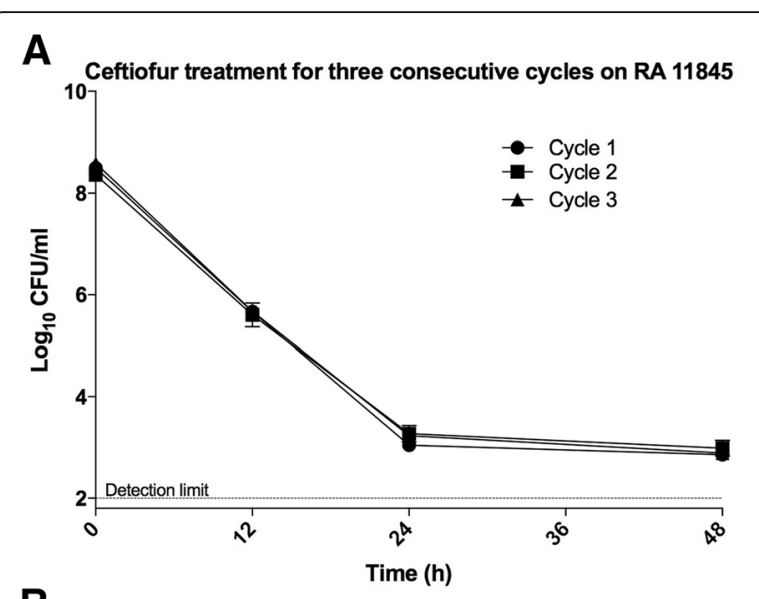

B

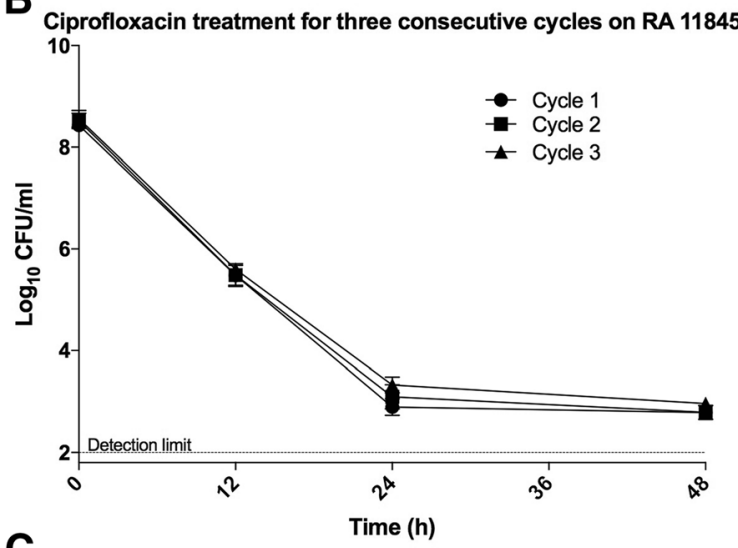

C

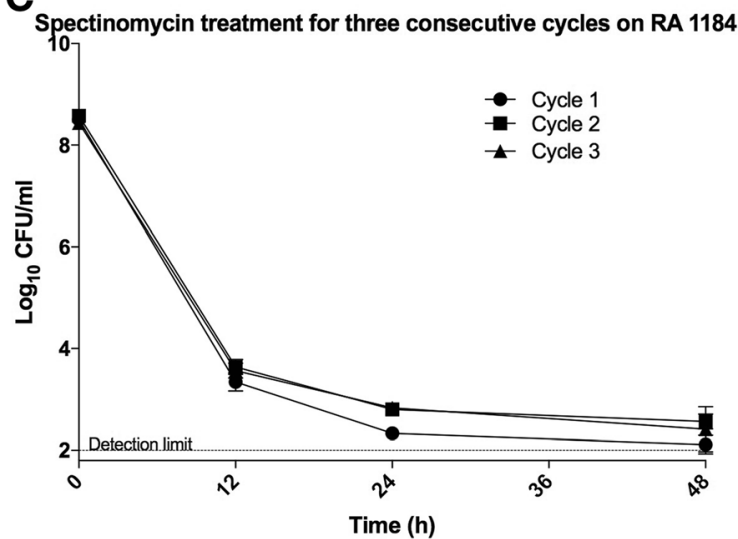

Fig. 2 Examination for heritability of persistence. Exponential growing cells of RA 11845 were challenged with 80-fold MIC of ceftiofur (a), ciprofloxacin (b), or spectinomycin (c) for $48 \mathrm{~h}$ in three consecutive cycles. The detection limit was $10^{2} \mathrm{CFU} / \mathrm{ml}$. Each spot showing on the figure was the mean of three biological replicates. The error bars represent the standard deviation of the mean

Persisters of RA TQ3 were eradicated by a combination of two or three antibiotics

To better understand the interactions between drugs, we performed a checkerboard assay. As shown in Table 1, the FICI values from all drug combinations for each RA strain were of $>0.5$ to $\leq 1$, indicating an additive effect 
between any two drugs. We therefore hypothesize that a combination of any two drugs, each at $\mathrm{C}_{\max }$, might reduce the persister level of RA TQ3. To our surprise, spectinomycin not just killed the non-tolerant cells but eliminate the persister cells of RA TQ3 within $21 \mathrm{~h}$, when used in concert with ciprofloxacin or ceftiofur. Similar results were observed when exponential growing population of RA TQ3 was challenged with drug combination of ceftiofur and ciprofloxacin, or treated with three antimicrobials simultaneously, except that the drug combination of ceftiofur and ciprofloxacin abolished the persisters of RA TQ3 within $36 \mathrm{~h}$, whereas combination of all three test drugs eliminated the persisters of RA TQ3 within $18 \mathrm{~h}$ (Fig. 3).

\section{Discussion}

It has been suggested that Riemerella anatipestife (RA) can form biofilms on certain criteria [27]. This phenomenon led us to hypothesize that persister cells might exist in RA population, because, persisters are produced in biofilms [20]. In the present study, we confirmed the presence of persister cells in RA Population. The antibiotics we used to detect persister cells from exponential growing population of RA reference strain (RA 11845) or RA clinical isolate (RA TQ3) were ceftiofur, ciprofloxacin and spectinomycin, respectively. Ceftiofur, a veterinary antibiotic, has been approved for food animal use in the United States and Europe [28]. As a third generation of cephalosporin, ceftiofur has similar bactericidal mechanism to all $\beta$-lactam antibiotics which disrupts the integrity of bacterial cell wall by inhibiting the catalytic activity of penicillin-binding proteins (PBPs) $[28,29]$. Our results suggested a strong tolerance of RA TQ3 cells to ceftiofur at 80-fold MIC (Fig. 1a). This is unexpected, because, the cells of RA reference strain (RA 11845) was sensitive to ceftiofur at a similar dosage (Fig. 1a). Such robust tolerance of RA TQ3 cells to ceftiofur is probably due to its clinical origin, given that a clinical isolate of Klebsiella pneumoniae presented a higher tolerance to some of tested antimicrobials in comparison to the laboratory reference strain [30].

Drugs of quinolone family, such as ciprofloxacin and norfloxacin, are capable of binding to the DNA complex of type IIA topoisomerases, DNA gyrase (GyrA:GyrB) or topoisomerase IV (topo IV) (ParC:ParE), resulting in double-strand DNA breaking and cell death [31, 32]. In vitro assessment of a collection of quinolones against a dozen of veterinary pathogens indicated that ciprofloxacin was the most active quinolone against Mycoplasma hyopneumoniae, Bordetella bronchiseptica, Pasteurella multocida, and Haemophilus pleuropneumoniae [33]. Our data suggested that the bactericidal effect of ciprofloxacin on RA was poor in the first few hours; the cell number of RA TQ3 dropped slowly, irrespective of dosage. Such phenomenon was more evident on RA 11845 where the cell number hardly changed during the initial $6 \mathrm{~h}$ of exposure (Fig. 1b). This "delayed killing effect" of ciprofloxacin was also presented in certain strains of Klebsiella pneumoniae and Yersinia pestis [34, 35]. But the actual cause remains to be elucidated.

Spectinomycin has been traditionally defined as a bacteriostatic agent, working by destabilizing the binding of peptidyl-tRNA to ribosome $[29,36]$. In this study,

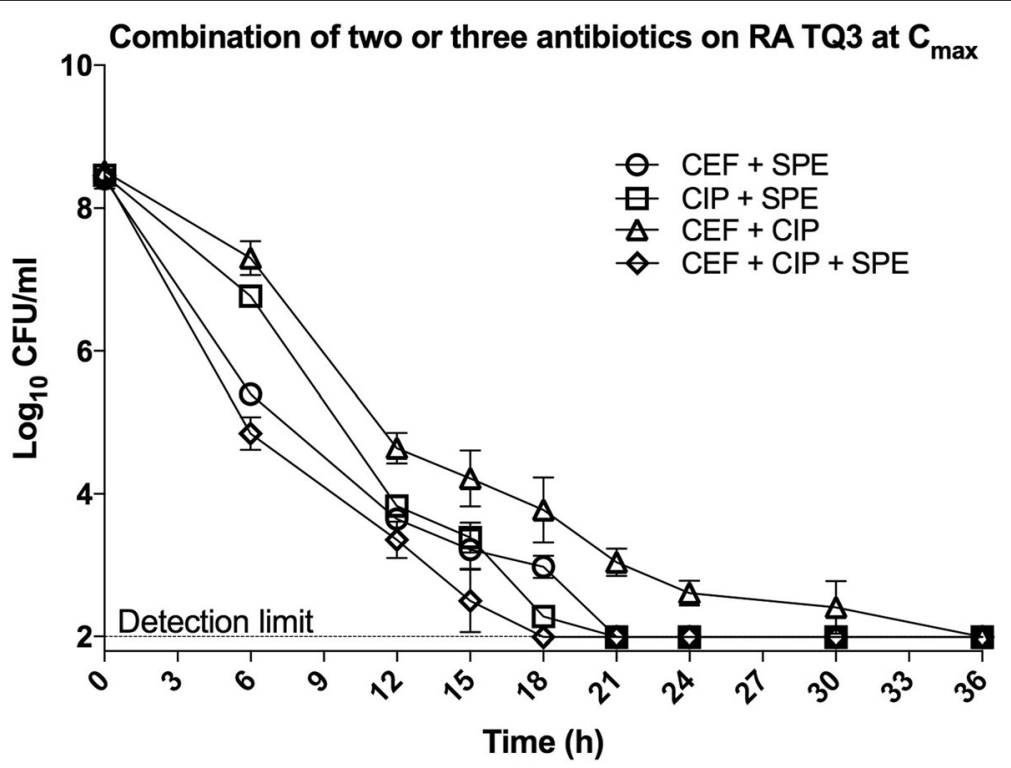

Fig. 3 Time-dependent killing by combining two or three antibiotics at respective $C_{\text {max }}$ against RA TQ3. The detection limit was $10^{2}$ CFU/ml. Each spot showing on the figure was the mean of three biological replicates. The error bars represent the standard deviation of the mean 
spectinomycin destroyed $99.9 \%$ of cells within $12 \mathrm{~h}$ when applied to exponential growing culture of RA 11845 or RA TQ3 (Fig. 1c). Such bactericidal action of spectinomycin was not limited to RA, but on Neisseria gonorrhoeae as well [37]. It was demonstrated that spectinomycin showed a higher binding affinity to the $30 \mathrm{~S}$ ribosomal proteins of Neisseria gonorrhoeae than that to those of $E$ coli where it presented a bacteriostatic activity [38]. Perhaps, a similar high avidity of spectinomycin to the $30 \mathrm{~S}$ ribosomal proteins of RA might exist which led to the bactericidal action.

As $\mathrm{C}_{\max }$ representing the highest concentration of a drug that can reach in serum/plasma after a single dose, we sought to address whether a test antibiotic can abolish all cells of RA at $C_{\max }$. Our data suggested that the $\mathrm{C}_{\max }$ of ceftiofur wiped out the non-tolerant cells of RA TQ3 rapidly and left a steady population of persister cells (Fig. 1a). This is unanticipated, because a concentration of ceftiofur at 80-fold MIC was unable to eradicate even the non-tolerant cells of RA TQ3 (Fig. 1a). It appears that the non-tolerant cells of RA TQ3 can endure a degree of ceftiofur, but succumbs to a high concentration of ceftiofur at $\mathrm{C}_{\max }(13.1 \mu \mathrm{g} / \mathrm{ml}, 210$-fold MIC, Table 1). In contrast, the $C_{\max }$ of ceftiofur eliminated all bacterial cells of RA 11845 within 48 h of treatment. We attributed this to the following reasons: the high concentration of ceftiofur at $C_{\max }(13.1 \mu \mathrm{g} / \mathrm{ml}$, 420-fold MIC, Table 1) improved its own bactericidal activity; the reference strain (RA 11845) was more vulnerable to ceftiofur, given that 10- and 80-fold MIC of ceftiofur killed the sensitive cells of RA 11845 within $24 \mathrm{~h}$ but failed to eliminate the non-tolerant cells of RA TQ3 during the same period of time (Fig. 1a). Clinically, the concentration of a drug was fluctuated in serum/ plasma. Therefore, the true effect of an antimicrobial was anticipated to be lower than that at a constant $C_{\max }$. Moreover, we cannot expect to destroy persisters by increasing the dosage of a drug progressively. Because, with an increased serving dosage, the toxicity of a drug also increased. As different antibiotics that have different modes of action, we wondered whether combining two or three antimicrobials could abolish the RA persisters at $\mathrm{C}_{\max }$, since several reports has demonstrated that drug combination could impair or even eradicate the persisters [39-41]. Our data indicated that the persisters of RA TQ3 were eliminated completely by a combination of any two or three drugs. In a previous study, Ramandeep and colleagues [39] have revealed two types of persisters: one-drug tolerant persisters, and multidrug tolerant persisters. The one-drug tolerant persisters are capable of enduring one drug but are not cross-tolerant to others drugs. The multidrug tolerant persisters are able to endure multiple antimicrobials. Apparently, the RA persisters are one-drug tolerant persisters and not multidrug tolerant persisters, because they cannot survive in the presence of multiple antibiotics.

The host environment is more complicated than in vitro conditions. Except the fluctuation of drug concentration in serum/plasma that we mentioned above, other factors such as the host-produced substances and local $\mathrm{pH}$ also affect the action of an antibiotic. Therefore, we cannot conclude that drug combination is more effectiveness against RA persisters comparing to a single antimicrobial in vivo. However, this study presents a potential solution to eradicate persisters, not only for RA, but for other zoonotic pathogens.

\section{Conclusion}

In this study, we confirmed the presence of drug tolerant persisters in RA population. The test antibiotics, including ceftiofur, ciprofloxacin and spectinomycin, cannot eradicate the persisters of RA TQ3 alone at $\mathrm{C}_{\max }$. However, a combination of two or three antibiotics eliminated the persisters of RA TQ3 completely at $\mathrm{C}_{\max }$. Our investigations provide a way to wipe out the drug tolerant persisters, which is helpful for the clinical treatment of RA infection in poultry.

\section{Methods}

\section{Antibiotics, bacterial strains and culture conditions}

Ceftiofur hydrochloride was purchased from Aladdin (Shanghai, China). Ciprofloxacin hydrochloride and spectinomycin were obtained from Sigma-Aldrich (St. Louis, MO, USA). All antibiotics used were of analytical standard (purity > 99.9\%). A reference strain, RA 11845, was purchased from American Type Culture Collection (ATCC). A clinical isolate, RA TQ3, was a kind gift from Dr. Lin at Sichuan Entry-Exit Inspection and Quarantine Bureau. The RA TQ3, sensitive to all test antimicrobials, was isolated from a batch of imported breeding chickens in 2016. Strains were routinely grown on trypticase soy agar (TSA) with 5\% sheep blood. Due to the growth deficiency of RA in Mueller-Hinton broth (MHB), trypticase soy broth (TSB) was used to culture cells and determine the MICs of antibiotics.

\section{The MIC measurement}

The MIC measurement was conducted as described previously [42]. In brief, all wells in each row of a 96-well plate (Costar 3599, Corning, New York, USA) were filled with $100 \mu \mathrm{l}$ of TSB medium, except the first one, which was filled with $200 \mu$ l of TSB medium to serve as a blank control. $100 \mu \mathrm{l}$ of fresh medium containing 32-fold the expected MIC of an antimicrobial agent was then added to the second well, followed by a 2-fold serial dilution from the second well to the 11th well. The last well contained no drugs was served as a positive control of growth. Overnight culture of RA 11845 or RA TQ3 was 
inoculated in $1 \mathrm{ml}$ of fresh TSB in a ratio of 1:10, and incubated at $37^{\circ} \mathrm{C}$ with shaking at $180 \mathrm{rev} / \mathrm{min}$. When the optical density (OD) reached at $\sim 0.2\left(10^{9} \mathrm{CFU} / \mathrm{ml}\right)$, $100 \mu \mathrm{l}$ culture was diluted at 1:1000 in fresh TSB medium. $100 \mu \mathrm{l}$ of the diluted culture containing $10^{5} \mathrm{CFU} / \mathrm{ml}$ of cells were inoculated from the second well to the 12th well. The plate was capped and incubated at $37^{\circ} \mathrm{C}$ for $18 \mathrm{~h}$, OD was then measured by a Multiskan GO Spectrophotometer (ThermoFisher Scientific, San Jose, CA, USA). The MIC was determined as the lowest concentration of a respective antibiotic which inhibited the growth of cells. The MIC measurement for each drug was repeated three times.

\section{Checkerboard assay}

The interactions between antibiotics were evaluated by the checkerboard assay $[43,44]$. The concentration range of each antibiotic in each drug combination was from 2 -fold to $1 / 16$-fold the MIC. The turbidity was checked after $18 \mathrm{~h}$ of incubation at $37^{\circ} \mathrm{C}$. The fractional inhibitory concentration index (FICI) was calculated using the following formula: $\mathrm{FICI}=\mathrm{MIC}_{\mathrm{A}+\mathrm{B}} / \mathrm{MIC}_{\mathrm{A}}+\mathrm{MIC}_{\mathrm{B}+\mathrm{A}}$ $/ \mathrm{MIC}_{\mathrm{B}}$. The $\mathrm{MIC}_{\mathrm{A}}$ and $\mathrm{MIC}_{\mathrm{B}}$ represent the $\mathrm{MIC}$ of drug $\mathrm{A}$ and drug $\mathrm{B}$ alone, respectively. The $\mathrm{MIC}_{\mathrm{A}+\mathrm{B}}$ is the MIC of drug $A$ in the presence of drug $B$, and vice versa for $\mathrm{MIC}_{\mathrm{B}+\mathrm{A}}$. The FICI values were interpreted as follows: synergy, FICI $\leq 0.5$; additivity, FICI $>0.5$ to $\leq 1$; no interaction, FICI $>1$ to $\leq 4$; antagonism, FICI $>4$. For each drug combination, the checkerboard assay was repeated three times.

\section{Determination of persister level}

The persister level of each RA strain was evaluated by a time-dependent killing experiment, using three antibiotics (ceftiofur, ciprofloxacin, and spectinomycin). Briefly, $100 \mu$ l overnight culture of RA $11845(\sim 4.5 \times$ $\left.10^{9} \mathrm{CFU} / \mathrm{ml}\right)$ or RA TQ3 $\left(\sim 3.8 \times 10^{9} \mathrm{CFU} / \mathrm{ml}\right)$ was inoculated in $100 \mathrm{ml}$ (1:1000) of fresh TSB medium, and incubated at $37^{\circ} \mathrm{C}$ with shaking at $180 \mathrm{rev} / \mathrm{min}$. The culture was divided into aliquots of $25 \mathrm{ml}$ when an $\mathrm{OD}_{600}$ value arrived at $0.1 \sim 0.2\left(1.7 \times 10^{8} \sim 3.8 \times 10^{8} \mathrm{CFU} / \mathrm{ml}\right)$. A single antibiotic was added to the aliquots of culture in a final concentration of 10-, 20-, 40- and 80-fold the MIC, respectively. Cultures were incubated as described above. At designated time points, $1 \mathrm{ml}$ sample from each culture was withdrawn, washed and resuspended in an equal volume of $1.0 \%(w / v)$ saline solution. Cell suspension was then submitted to bacterial numeration using standard plate counting method. The persister level was determined by the number of survivors on the plateau of the biphasic killing curves. All experiments were performed with three independent biological replicates.

In clinical practice, the serum/plasma concentration of an antimicrobial reaches the peak $\left(\mathrm{C}_{\max }\right)$ sometime post administration. Therefore, it is of great importance to evaluate the persister level of each RA strain under the treatment of a single antibiotic at $C_{\max }$ due to the potential of persisters to cause a relapse. For this purpose, we performed a similar time-dependent killing experiment as mentioned above, except that the final concentration of a drug in culture was adjusted to $C_{\max }$. Due to the shortage of pharmacokinetic parameters of ciprofloxacin and spectinomycin in ducks, the $\mathrm{C}_{\max }$ of these antibiotics for chickens are adopted instead. The $C_{\max }$ values of all tested antibiotics are listed in Table 2.

\section{Inheritability of drug tolerance for persisters}

An aliquot of exponential phase culture of RA 11845 was exposed to 80-fold MIC of ceftiofur, ciprofloxacin, or spectinomycin for $48 \mathrm{~h}$. At designated time points, cells were harvested, washed, and counted on non-selective agar (TSA with $5 \%$ sheep blood). At the end of each treatment, $100 \mu \mathrm{l}$ culture was withdrawn, washed and inoculated in $10 \mathrm{ml}$ of fresh TSB medium. After incubated at $37^{\circ} \mathrm{C}$ overnight with shaking at $180 \mathrm{rev} / \mathrm{min}$, cells were diluted at 1:1000 in fresh TSB medium and regrew to exponential phase to complete the first cycle. Cells were then challenged with antibiotics as described above, followed by cell numeration at designated time points. The procedure was repeated for three consecutive cycles.

\section{The persister level of RA TQ3 under the treatment of a combination of two or three drugs}

A batch of exponential phase culture of RA TQ3 was challenged with a combination of two or three antimicrobials. The final concentration of each antibiotic in cell culture was adjusted to $\mathrm{C}_{\max }$. At designated time points, culture samples were taken, washed, and plated after serial dilution for CFUs. The level of persisters was again evaluated by the number of survivors showing on the plateau of the killing curves. For each drug combination, the experiment was repeated three times.

Table 2 The peak serum/plasma concentration $\left(C_{\max }\right)$ of antimicrobial agents

\begin{tabular}{lllll}
\hline Antibiotics & Route of administration & Avian species & $C_{\max }(\mu \mathrm{g} / \mathrm{ml})$ & Reference \\
\hline Ceftiofur & Subcutaneous injection & American black ducks & 13.1 & {$[45]$} \\
Ciprofloxacin & Oral administration & broiler chickens & 4.67 & [46] \\
Spectinomycin & Intramuscular injection & Broiler chickens & 152.76 & {$[47]$} \\
\hline
\end{tabular}




\section{Additional file}

Additional file 1: Table S1. Exponentially grown RA population was exposed to 20- and 40-fold the MIC of ceftiofur (A), ciprofloxacin (B) and spectinomycin $(C)$, respectively. RE presents RA reference strain, RA 11845; TQ3 presents RA clinical isolate, RA TQ3. The detection limit was $10^{2}$ CFU/ $\mathrm{ml}$. Each spot showing on the figure was the mean of three biological replicates. The error bars represent the standard deviation of the mean. (TIF 940 kb)

\section{Abbreviations}

CEF: Ceftiofur; CFU: Colony-forming unit; CIP: Ciprofloxacin; $C_{\text {max: }}$ Maximum serum/plasma concentration; FICl: Fractional inhibitory concentration index; MHB: Mueller-Hinton broth; MIC: Minimum inhibitory concentration; OD600: Optical density at $600 \mathrm{~nm}$; RA: Riemerella anatipestifer; SPE: Spectinomycin; TSA: Trypticase soy Agar; TSB: Trypticase soy broth

\section{Acknowledgements}

We gratefully acknowledge Dr. Lin at Sichuan Entry-Exit Inspection and Quarantine Bureau for RA clinical isolate TQ3. We also thank Prof. Yanxia Wu for experimental design and guide.

\section{Funding}

This work is supported by Sichuan Science \& Technology Department Foundation (No. 2017JY0240), China Postdoctoral Science Foundation (No. 2016 M602691), National Mega Projects of Science and Technology in 13th 5-Year Plan of China: Technical Platform for Communicable Disease Surveillance (No. 2017ZX10103010-002), Young Scholar Research Grants of Sichuan University (No. 2016SCU11005) and Natural Science Foundation of China (No. 31570924). The funding bodies had no role in the design of the study and collection, analysis and interpretation of data and in writing the manuscript.

\section{Availability of data and materials}

All data generated or analyzed during this study are included in this published article.

\section{Authors' contributions}

The research idea was derived from $\Pi$ and $\mathrm{YW}$. $\Pi$, YW, CW, and XP designed all experiments. $\Pi, Y L, H L, Q G$ and $H Z$ performed the experiments. $\Pi$ analyzed the data. TT and YW wrote this paper. All authors read and approved the final manuscript.

\section{Ethics approval and consent to participate}

Not applicable

\section{Consent for publication}

Not applicable

\section{Competing interests}

The authors declare that they have no competing interests.

\section{Publisher's Note}

Springer Nature remains neutral with regard to jurisdictional claims in published maps and institutional affiliations.

\section{Author details}

${ }^{1}$ Department of Public Health Laboratory Sciences, West China School of Public Health, Sichuan University, 16\#, Section 3, South Renmin Road, Chengdu, Sichuan 610031, People's Republic of China. ${ }^{2}$ Sichuan Entry-Exit Inspection and Quarantine Bureau, Chengdu, Sichuan, People's Republic of China. ${ }^{3}$ Institute of Preventive Veterinary Medicine, Sichuan Agricultural University, Chengdu, Sichuan, People's Republic of China.
Received: 20 December 2017 Accepted: 4 October 2018

Published online: 19 October 2018

\section{References}

1. Tang T, Gao Q, Barrow P, Wang M, Cheng A, Jia R, Zhu D, Chen S, Liu M, Sun $\mathrm{K}$, et al. Development and evaluation of live attenuated Salmonella vaccines in newly hatched duckings. Vaccine. 2015;33(42):5564-71.

2. Subramaniam S, Huang B, Loh H, Kwang J, Tan HM, Chua KL, Frey J. Characterization of a predominant immunogenic outer membrane protein of Riemerella anatipestifer. Clin Diagn Lab Immunol. 2000;7(2):168-74.

3. Huang B, Kwang J, Loh H, Frey J, Tan HM, Chua KL. Development of an ELISA using a recombinant $41 \mathrm{kDa}$ partial protein (P45N') for the detection of Riemerella anatipestifer infections in ducks. Vet Microbiol. 2002;88(4):339-49.

4. Chang CF, Lin WH, Yeh TM, Chiang TS, Chang YF. Antimicrobial susceptibility of Riemerella anatipestifer isolated from ducks and the efficacy of ceftiofur treatment. J Vet Diagn Investig. 2003;15(1):26-9

5. Li J, Tang Y, Gao J, Huang C, Ding M. Riemerella anatipestifer infection in chickens. Pak Vet J. 2011;31:65-9.

6. Chen YP, Tsao MY, Lee SH, Chou CH, Tsai HJ. Prevalence and molecular characterization of chloramphenicol resistance in Riemerella anatipestifer isolated from ducks and geese in Taiwan. Avian Pathol. 2010;39(5):333-8.

7. Chen YP, Lee SH, Chou CH, Tsai HJ. Detection of florfenicol resistance genes in Riemerella anatipestifer isolated from ducks and geese. Vet Microbiol. 2012;154(3-4):325-31.

8. Rubbenstroth D, Ryll M, Behr KP, Rautenschlein S. Pathogenesis of Riemerella anatipestifer in turkeys after experimental mono-infection via respiratory routes or dual infection together with the avian metapneumovirus. Avian Pathol. 2009;38(6):497-507.

9. Hu Q, Tu J, Han X, Zhu Y, Ding C, Yu S. Development of multiplex PCR assay for rapid detection of Riemerella anatipestifer, Escherichia coli, and Salmonella enterica simultaneously from ducks. J Microbiol Methods. 2011; 87(1):64-9.

10. Kardos G, Nagy J, Antal M, Bistyak A, Tenk M, Kiss I. Development of a novel PCR assay specific for Riemerella anatipestifer. Lett Appl Microbiol. 2007; 44(2):145-8

11. Wang XP, Zhu DK, Wang MS, Cheng AC, Jia RY, Chen S, Chen XY, Tang T, Development and application of specific polymerase chain reaction assay targeting the gyrB gene for rapid detection of Riemerella anatipestifer. Poult Sci. 2012;91(10):2450-3.

12. Crasta KC, Chua KL, Subramaniam S, Frey J, Loh H, Tan HM. Identification and characterization of CAMP cohemolysin as a potential virulence factor of Riemerella anatipestifer. J Bacteriol. 2002;184(7):1932-9.

13. Hu Q, Han X, Zhou X, Ding C, Zhu Y, Yu S. OmpA is a virulence factor of Riemerella anatipestifer. Vet Microbiol. 2011;150(3-4):278-83.

14. Pathanasophon P, Sawada T, Tanticharoenyos T. New serotypes of Riemerella anatipestifer isolated from ducks in Thailand. Avian Pathol. 1995; 24(1):195-9.

15. Pathanasophon P, Phuektes $P$, Tanticharoenyos T, Narongsak W, Sawada T. A potential new serotype of Riemerella anatipestifer isolated from ducks in Thailand. Avian Pathol. 2002;31(3):267-70.

16. Ryll M, Hinz KH. Exclusion of strain 670/89 as type strain for serovar 20 of Riemerella anatipestifer. Berl Munch Tierarztl Wochenschr. 2000;113(2):65-6.

17. Fulton RM, Rimler RB. Epidemiologic investigation of Riemerella anatipestifer in a commercial duck company by serotyping and DNA fingerprinting. Avian Dis. 2010:54(2):969-72.

18. Huang C-H, Li J-X, Huang W, Li X, Yang P-D, Yang Y-H. Dynamic epidemiological investigation of riemerella anatipestifer isolated from ducklings in Chongqing and Sichuan. Chin J Prev Vet Med. 2007;1:016

19. Hu Q, Zhang Z, Miao J, Liu Y, Liu X, Ding C. Epidemiologic investigation of Riemerella anatipestifer infection in ducks in Jiangsu and Anhui provinces. Chin J of Vet Sci Technol. 2001;31(8):12-3.

20. Zhong CY, Cheng AC, Wang MS, Zhu DK, Luo QH, Zhong CD, Li L, Duan Z. Antibiotic susceptibility of Riemerella anatipestifer field isolates. Avian Dis. 2009:53(4):601-7.

21. Sun N, Liu JH, Yang F, Lin DC, Li GH, Chen ZL, Zeng ZL. Molecular characterization of the antimicrobial resistance of Riemerella anatipestifer isolated from ducks. Vet Microbiol. 2012;158(3-4):376-83.

22. Shah D, Zhang Z, Khodursky A, Kaldalu N, Kurg K, Lewis K. Persisters: a distinct physiological state of E coli. BMC Microbiol. 2006;6:53. 
23. Lewis K. Persister cells, dormancy and infectious disease. Nat Rev Microbiol. 2007;5(1):48-56.

24. Keren I, Kaldalu N, Spoering A, Wang Y, Lewis K. Persister cells and tolerance to antimicrobials. FEMS Microbiol Lett. 2004:230(1):13-8.

25. Maisonneuve E, Gerdes K. Molecular mechanisms underlying bacterial persisters. Cell. 2014;157(3):539-48.

26. Van den Bergh B, Fauvart M, Michiels J. Formation, physiology, ecology, evolution and clinical importance of bacterial persisters. FEMS Microbiol Rev. 2017:41(3):219-51.

27. Hu Q, Han X, Zhou X, Ding S, Ding C, Yu S. Characterization of biofilm formation by Riemerella anatipestifer. Vet Microbiol. 2010;144(3-4):429-36.

28. Hornish RE, Kotarski SF. Cephalosporins in veterinary medicine - ceftiofur use in food animals. Curr Top Med Chem. 2002;2(7):717-31.

29. Kohanski MA, Dwyer DJ, Collins JJ. How antibiotics kill bacteria: from targets to networks. Nat Rev Microbiol. 2010;8(6):423.

30. Ren H, He X, Zou X, Wang G, Li S, Wu Y. Gradual increase in antibiotic concentration affects persistence of Klebsiella pneumoniae. J Antimicrob Chemother. 2015;70(12):3267-72.

31. Kohanski MA, Dwyer DJ, Hayete B, Lawrence CA, Collins JJ. A common mechanism of cellular death induced by bactericidal antibiotics. Cell. 2007; 130(5):797-810.

32. Wohlkonig A, Chan PF, Fosberry AP, Homes P, Huang J, Kranz M, Leydon VR, Miles TJ, Pearson ND, Perera RL, et al. Structural basis of quinolone inhibition of type IIA topoisomerases and target-mediated resistance. Nat Struct Mol Biol. 2010;17(9):1152-3.

33. Hannan $\mathrm{P}, \mathrm{O}$ 'hanlon $\mathrm{P}$, Rogers $\mathrm{N}$. In vitro evaluation of various quinolone antibacterial agents against veterinary mycoplasmas and porcine respiratory bacterial pathogens. Res Vet Sci. 1989:46(2):202-11.

34. Grillon A, Schramm F, Kleinberg M, Jehl F. Comparative activity of ciprofloxacin, levofloxacin and Moxifloxacin against Klebsiella pneumoniae, Pseudomonas aeruginosa and Stenotrophomonas maltophilia assessed by minimum inhibitory concentrations and time-kill studies. PLoS One. 2016; 11(6):e0156690.

35. Lemaitre N, Ricard I, Pradel E, Foligne B, Courcol R, Simonet M, Sebbane F. Efficacy of ciprofloxacin-gentamicin combination therapy in murine bubonic plague. PLoS One. 2012;7(12):e52503.

36. Davis BD. Mechanism of bactericidal action of aminoglycosides. Microbiol Rev. 1987;51(3):341-50.

37. Ward ME. The bactericidal action of spectinomycin on Neisseria gonorrhoeae. J Antimicrob Chemother. 1977;3(4):323-9.

38. Maness MJ, Foster GC, Sparling PF. Ribosomal resistance to streptomycin and spectinomycin in Neisseria gonorrhoeae. J Bacteriol. 1974;120(3):1293-9.

39. Singh R, Barry CE 3rd, Boshoff HI. The three RelE homologs of Mycobacterium tuberculosis have individual, drug-specific effects on bacterial antibiotic tolerance. J Bacteriol. 2010;192(5):1279-91.

40. Feng J, Auwaerter PG, Zhang Y. Drug combinations against Borrelia burgdorferi persisters in vitro: eradication achieved by using daptomycin, cefoperazone and doxycycline. PLoS One. 2015;10(3):e0117207.

41. Chua SL, Yam JK, Hao P, Adav SS, Salido MM, Liu Y, Givskov M, Sze SK, Tolker-Nielsen T, Yang L. Selective labelling and eradication of antibiotic tolerant bacterial populations in Pseudomonas aeruginosa biofilms. Nat Commun. 2016;7:10750.

42. Wu Y, Vulic M, Keren I, Lewis $K$. Role of oxidative stress in persister tolerance. Antimicrob Agents Chemother. 2012;56(9):4922-6.

43. Dosler S, Karaaslan E, Alev Gerceker A. Antibacterial and anti-biofilm activities of melittin and colistin, alone and in combination with antibiotics against gram-negative bacteria. J Chemother. 2016;28(2):95-103.

44. Jayaraman P, Sakharkar MK, Lim CS, Tang TH, Sakharkar KR. Activity and interactions of antibiotic and phytochemical combinations against Pseudomonas aeruginosa in vitro. Int J Biol Sci. 2010;6(6):556-68.

45. Hope KL, Tell LA, Byrne BA, Murray S, Wetzlich SE, Ware LH, Lynch W, Padilla $L R$, Boedeker NC. Pharmacokinetics of a single intramuscular injection of ceftiofur crystalline-free acid in American black ducks (Anas rubripes). Am J Vet Res. 2012;73(5):620-7.

46. Atta AH, Sharif L. Pharmacokinetics of ciprofloxacin following intravenous and oral administration in broiler chickens. J Vet Pharmacol Ther. 1997:20(4): 326-9.

47. Abu-Basha EA, Gehring R, Albwa'neh SJ. Pharmacokinetics and bioavailability of spectinomycin after i.v., i.m., s.c. and oral administration in broiler chickens. J Vet Pharmacol Ther. 2007:30(2):139-44.

\section{Ready to submit your research? Choose BMC and benefit from:}

- fast, convenient online submission

- thorough peer review by experienced researchers in your field

- rapid publication on acceptance

- support for research data, including large and complex data types

- gold Open Access which fosters wider collaboration and increased citations

- maximum visibility for your research: over $100 \mathrm{M}$ website views per year

At BMC, research is always in progress.

Learn more biomedcentral.com/submissions 